\title{
Alternativas a la segregación escolar: estrategias desde la educación no formal
}

\section{Alma Arcelia Ramírez Íñiguez}

https://orcid.org/00oo-0003-3171-2417

Universidad Autónoma de Baja California,

México

alma.arcelia.ramirez.iniguez@uabc.edu.mx

\section{Resumen}

Este artículo analiza las estrategias, procesos y relaciones que se desarrollan en el ámbito no formal de la educación, para contribuir a resolver el problema de la segregación escolar en el contexto latinoamericano. Se identifica el vínculo entre segregación y exclusión y se exponen las caracteristicas de los procesos educativos no formales que coadyuvan a la inclusión educativa. Se realiza un análisis cualitativo de diversas experiencias educativas, en diferentes países de América Latina, que presentan alternativas a la escolarización y acciones para el desarrollo de competencias relevantes de los estudiantes. Los resultados muestran que la educación no formal es un ámbito que abre posibilidades de formación, que en la educación escolarizada se ven limitadas por su tendencia a reproducir la exclusión social.

\section{Palabras claves (Fuente: tesauro de la Unesco)}

Educación alternativa; educación no formal; integración escolar; pedagogía social; segregación escolar; sistemas educativos.

Recepción: 28/07/2020 | Envío a pares: 16/11/2020 | Aceptación por pares: 26/11/2020 | Aprobación: 9/12/2020 


\title{
Alternatives to School Segregation: Strategies from Nonformal Education
}

\begin{abstract}
This article discusses the strategies, processes, and relationships in nonformal education to help solve school segregation in the Latin American context. It identifies the link between segregation and exclusion and reveals the characteristics of nonformal educational processes that contribute to educational inclusion. We carried out a qualitative analysis of various educational experiences in Latin American countries having alternatives to schooling and actions to develop students'relevant skills. The results show that nonformal education opens up training possibilities, limited in school-based education by its tendency to reproduce social exclusion.
\end{abstract}

\section{Keyword (Source: Unesco Thesaurus)}

Alternative education; nonformal education; school integration; social pedagogy; school segregation; educational systems. 


\section{Alternativas para a segregação escolar: estratégias a partir da educação não formal}

\section{Resumo}

Este artigo analisa as estratégias, processos e relações que se desenvolvem no âmbito não formal da educação, para contribuir com a resolução do problema da segregação escolar no contexto latino-americano. Identifica-se o vínculo entre segregação e exclusão e expõem-se as características dos processos educacionais não formais que contribuem para a inclusão educacional. Realiza-se uma análise qualitativa de diversas experiências educacionais, em diferentes países da América Latina, que apresentam alternativas para a escolarização e ações para o desenvolvimento de competências relevantes dos estudantes. Os resultados mostram que a educação não formal é um âmbito que abre possibilidades de formação, que na educação escolarizada se veem limitadas por sua tendência a reproduzir a exclusão social.

\section{Palavras-chave (Fonte: tesauro da Unesco)}

Educação alternativa; educação informal; integração escolar; pedagogia social; segregação escolar; sistemas educacionais. 
En las últimas décadas, la región latinoamericana se ha caracterizado por una profunda desigualdad socioeconómica que se reproduce en los sistemas educativos. Al respecto, algunos estudios (Krüger, 2019) evidencian que hay un estrecho vínculo entre la segregación escolar y los altos niveles de pobreza y la precaria inversión en educación, así como la tendencia a incrementar la escolarización privada, todo lo cual agudiza la segmentación entre quienes pueden acceder a una educación de calidad y quienes tienen pocas alternativas de formación. Esta relación se manifiesta de manera importante en el logro desigual de los aprendizajes fundamentales para la vida (Villalta y Assael, 2018), donde los sectores socialmente más desfavorecidos difícilmente reciben una escolarización que propicie el desarrollo de las competencias básicas para desenvolverse plenamente en las sociedades.

Sin bien América Latina ha tenido un importante avance en la expansión de la cobertura en los niveles básicos del sistema educativo, este crecimiento ha sido desigual en el alcance de los aprendizajes socialmente relevantes por parte de todas las personas que, a pesar de estar integradas en el sistema escolar, no han conseguido este desarrollo. Dicha situación representa la expresión más contundente de la segregación escolar en el contexto latinoamericano. Las investigaciones al respecto (Murillo y Martínez, 2017; Murillo, 2016; Vázquez, 2016) coinciden en que este es un problema que demuestra una exclusión de la calidad educativa asociada al nivel socioeconómico, al origen étnico-cultural y a la diversidad funcional, aspectos que las escuelas no están atendiendo, con lo que se crean importantes rezagos entre la población, no solo en el logro de los aprendizajes, sino también en el efectivo ejercicio de los derechos ciudadanos. Además, señalan que la segregación se manifiesta en una jerarquización de la población en relación con sus oportunidades educativas, donde los sectores socialmente más desaventajados se concentran en las escuelas con los resultados educativos más bajos.
Particularmente, en América Latina la población indígena, la inmigrante y aquella con niveles socioeconómicos inferiores a la media del bienestar son los que más padecen esta problemática (Murillo y Martínez, 2017; Murillo, 2016). Por lo tanto, además de afectar la adquisición de los aprendizajes necesarios para el desarrollo personal y social, la segregación escolar profundiza los problemas de discriminación y exclusión, produce grupos cada vez más segmentados y ahonda la desigualdad (Murillo et al., 2018). Así, este fenómeno contribuye a agudizar la vulnerabilidad de la población menos privilegiada en un proceso interconectado entre la sociedad y la escuela.

En términos porcentuales, la segregación escolar expone la falta de inclusión -más allá de la integración escolar- de los sistemas educativos, donde solo $53 \%$ de los adolescentes latinoamericanos logra adquirir las competencias establecidas en los dos primeros niveles de las pruebas PISA (Programme for International Student Assessment), considerando igualmente que cerca de $20 \%$ de los jóvenes de 15 años no asiste a la escuela secundaria. De los que asisten, muchos presentan rezago o un alto riesgo de deserción (Krüger, 2019). A pesar de las reformas en la política educativa que la mayor parte de los países en América Latina han hecho en los últimos años, este panorama no se ha transformado. Estudios recientes (Murillo et al., 2018) demuestran que, aunque se observa cierta disminución de la segregación en algunos países, esta se presenta principalmente en los sectores de mayor nivel socioeconómico, acrecentándose en los de menos nivel. De este modo, la segregación es un problema que se da dentro de los sectores sociales y entre ellos.

No obstante, cabe señalar que algunos análisis enfatizan en las experiencias educativas donde la vulnerabilidad a la exclusión educativa y social no es una condicionante de la segregación, si se llevan a cabo acciones pertinentes (Villalta y Assael, 2018). Estas se han centrado en la creación de un clima favorable en el aula, en la participación del alum- 
nado en las actividades de clase y en el fomento del aprendizaje autónomo. Por lo tanto, se requiere profundizar en la reflexión crítica sobre los factores implicados en la mejora de la inclusión educativa. En este sentido, los análisis también toman en cuenta aquellos aspectos que en otros escenarios contribuyen a aminorar el problema de la segregación y sus consecuencias. Esto considerando el estrecho vínculo entre los procesos educativos y sociales, donde la segregación se visualiza como un problema interdependiente. Por ello, este trabajo reconoce que la atención a la segregación escolar y la mejora de la inclusión educativa son tareas complejas, por lo que se requiere analizar acciones que se desarrollan dentro y fuera de la escuela (Echeita, 2013).

Así, se analizan las contribuciones que se han realizado en la educación no formal como un ámbito que no solo contribuye al logro de los aprendizajes académicos de todas las personas y, por lo tanto, a la reducción de la segregación escolar, sino que tiene un papel fundamental en la formación de las competencias para la convivencia social. La segregación en América Latina exige contemplar la diversidad de iniciativas que puedan implementarse en múltiples áreas, por lo que este trabajo pretende aportar una perspectiva que contribuya a la necesaria reflexión sobre este problema y a la apertura de nuevas formas de solución.

\section{Revisión de la literatura}

La segregación escolar se ha definido como el resultado de un conjunto de políticas y procesos sociales que generan una distribución desigual del alumnado, principalmente por sus características étnicas, culturales y socioeconómicas, al punto de provocar que determinados grupos sean los únicos beneficiarios de una educación de calidad (Murillo y Belavi, 2018; Murillo y Martínez, 2017). Por lo tanto, se trata de un problema de justicia social y de falta de equidad en las oportunidades educativas.

En la segregación escolar se reconocen dos dimensiones que la caracterizan (Murillo y Martínez,
2019; Murillo et al., 2018): la de exposición, referente a la escolarización de grupos con características homogéneas, y la de uniformidad, a partir de la cual se distribuye de manera inequitativa al alumnado, donde algunos se ubican en escuelas que ofrecen mejores condiciones educativas y otros en aquellas que no logran los resultados esperados. En ambas dimensiones, el eje de la segregación es una clasificación dispar del alumnado en relación con las posibilidades que tiene para desarrollar sus aprendizajes. De acuerdo con algunos análisis (Córdoba et al., 2016), el lugar de residencia, los recursos de las familias para elegir la educación de sus miembros, así como la propia organización escolar son los principales factores que determinan la segregación y que profundizan la vulnerabilidad de los estudiantes más desaventajados.

En consecuencia, la segregación escolar repercute en la cohesión social, debilita la formación ciudadana y fomenta la exclusión (Murillo, 2016). En este sentido, las reflexiones sobre cómo resolver esta problemática deben partir de la identificación de aquellos aspectos que aminoran las condiciones que generan la segregación y que llevan a profundizar la exclusión social. Esto tomando en cuenta que la separación entre los diversos sectores dentro del ámbito educativo no solo es física sino también simbólica (Ascorra et al., 2016), de tal manera que entre los diferentes grupos no se retroalimenta la diversidad de referentes culturales, sociales y de saberes y ello debilita los lazos comunitarios, los cuales son necesarios para la construcción de sociedades más democráticas.

De esta manera, los procesos educativos son multidimensionales y propician la inclusión o la exclusión en relación con los siguientes aspectos (Krüger, 2019). El primero es la cobertura del sistema, la cual se centra en la asistencia a la escuela y en la continuidad de las trayectorias académicas; el segundo se refiere a la adquisición de las competencias que les permiten a las personas desenvolverse plenamente dentro de su sociedad; y el tercero se 
enfoca en el desarrollo de acciones que integran las dos primeras dimensiones para transformar la realidad de la segregación y vincular la educación con la inclusión social.

Al respecto, algunos estudios (Ortiz, 2015) han demostrado que el aprendizaje mejora cuando el alumnado de diferentes niveles socioeconómicos convive en los mismos espacios educativos. Esto se observa principalmente en los estudiantes con mayor desigualdad social. Sin embargo, aquellos que se ubican en los niveles medios y altos de bienestar también mejoran sus actitudes ante la diversidad y desarrollan el respeto y la tolerancia. En sintesis, una cobertura dirigida a crear grupos heterogéneos en la escuela contribuye a establecer la estructura fundamental para el logro de la inclusión educativa y social. Con esta visión, la segregación y la inclusión son conceptos que muestran los extremos de una misma realidad (Murillo y Duk, 2016). Por un lado, la segregación alude a la segmentación de los grupos sociales dentro del sistema educativo con una profunda desigualdad de oportunidades de desarrollo y, por otro, la inclusión permite comprender este problema y transformarlo hacia el logro de los aprendizajes por parte de todo el alumnado. Así, la integración de los estudiantes con diversas características en los mismos ambientes escolares es la base para la atención de la segregación, pero no es suficiente. Las dinámicas educativas, para ser inclusivas y transformar esta realidad, deben abarcar, además de la dimensión de cobertura, la correspondiente al logro de aprendizajes para el desarrollo de múltiples competencias.

En esta lógica, los procesos educativos se componen de factores escolares y del entorno (Ramírez, 2016). En relación con los primeros, se destaca el rol del profesorado, la organización escolar y curricular, así como la atención a la diversidad. Dentro de estos se encuentran la motivación del alumnado por los aprendizajes académicos, así como su reconocimiento, la atención a sus necesidades y la valoración de todos los aprendizajes que van logrando. Por lo tanto, las actividades educativas se evalúan sistemáti- camente para adaptarlas a las características de los estudiantes y para implementar los apoyos que exige su óptimo desarrollo, teniendo en cuenta que su participación es un componente clave. Por su parte, los factores del entorno se refieren al vínculo con las familias y la comunidad, así como a la implicación de otros agentes sociales.

De este modo, el problema de la segregación requiere mirar más allá de la propia escuela, considerando el trabajo integrado y cooperativo con otros organismos y con los ámbitos no formal e informal de la educación (Ramírez, 2014). En este sentido, se concibe la necesidad de una responsabilidad compartida para crear iniciativas que contribuyan a resolver los problemas escolares (Sabariego, 2009), partiendo de reconocer que estos son complejos y que exigen acciones coordinadas, desde distintas áreas, para su análisis y atención.

Específicamente, el ámbito de la educación no formal implica una idea comunitaria de la educación en donde todos los agentes involucrados se corresponsabilizan e involucran trayendo sus aportaciones con base en una comunicación bidireccional (Ferrer et al., 2006). Esto considerando que en este ámbito de la educación se adquieren aprendizajes que son tan relevantes como aquellos que se consiguen en los espacios escolares (Ortega, 2005). Así, el vínculo entre la educación escolar y la no formal posibilita el logro de diversos aprendizajes a partir de la coordinación, por lo que se atiende de manera integral la complejidad de las condiciones que propician o limitan los procesos de formación. Por consiguiente, se integran los esfuerzos de distintos actores para la solución de problemas como la segregación. Desde este enfoque, se concibe que las múltiples dimensiones que componen un fenómeno como este se resuelven más eficazmente trabajando en distintas áreas.

Por otro lado, la educación no formal se caracteriza por ser una alternativa a la escolarización, en un proceso de aprendizaje a lo largo de la vida (Olcott, 
2013), es decir, más allá de lo que ofrece el sistema educativo, sin que necesariamente conlleve una certificación que valide la formación recibida. Sin embargo, complementa la educación escolar para constituir procesos completos de formación. Por lo tanto, los espacios de educación no formal conforman una opción para transformar las dinámicas que fomentan la exclusión. Así, se establecen acciones colaborativas que articulan los esfuerzos desde dentro y fuera del espacio escolar para propiciar la inclusión.

De esta manera, el ámbito de la educación no formal coadyuva a la cohesión social, al fortalecer no solo los aprendizajes académicos (Chávez, 2013), sino también aquellos que contribuyen a la participación y la relación del alumnado con distintos referentes culturales y condiciones socioeconómicas, mediante la integración de diversas actividades (Cabrero y Valencia, 2019). En este sentido, se conjugan acciones que favorecen la eliminación de las barreras que limitan los aprendizajes, especialmente en el caso de los estudiantes con mayor desventaja social.

A partir de lo anterior, se destaca que las estrategias educativas que posibilitan la inclusión desde el ámbito no formal se caracterizan por los siguientes aspectos.

- $\quad$ Son procesos que tienen la finalidad de contribuir a la inclusión educativa y social, especialmente del alumnado más segregado dentro del sistema escolar, de tal manera que se enfocan a crear vínculos con los sectores de la educación formal y con otros agentes sociales para coordinar acciones que posibiliten el logro de los aprendizajes que mejoran la convivencia social.

- Se orientan al fortalecimiento de las competencias para desenvolverse plenamente en la sociedad. Por lo tanto, retroalimentan los aprendizajes académicos y generan otros que contribuyen a la formación integral del alumnado, apoyando una educación que prepara a las personas para ejercer sus derechos ciudadanos.
- Implican la participación tanto del alumnado como de las familias, la comunidad y otros agentes sociales, con el propósito de transformar la realidad de segregación por una que parte de las diversas perspectivas que se conjugan en los procesos educativos. En este sentido, se abren posibilidades de una educación más apegada a las condiciones de los integrantes del propio proceso a partir de sus aportaciones.

\section{Metodología de análisis}

La estrategia metodológica elegida para este estudio es el análisis de contenido cualitativo de diferentes experiencias latinoamericanas que documentan los factores intervinientes en procesos educativos no formales desarrollados en entornos de segregación escolar. Esta estrategia permitió un análisis sistemático de los artículos seleccionados a través de la categorización y la interpretación de sus significados (Nacak y Yücesoy, 2020). Desde el enfoque cualitativo, el uso de categorías posibilita la agrupación de elementos comunes de acuerdo con las unidades que conforman el contenido de las experiencias documentadas (López, 2002). Este proceso puede realizarse de manera deductiva, inductiva o mixta (Guix, 2008). En este trabajo, se optó por una categorización mixta, en la que, a partir de categorías teóricas, se identificaron los aspectos fundamentales de cada experiencia y se encontraron las relaciones entre ellas, lo que generó nuevas categorías a partir del vínculo entre las categorías teóricas y la información recopilada. Así, se reconocieron los factores de la educación no formal que favorecen la inclusión educativa y contrarrestan la segregación escolar en los casos analizados.

La búsqueda de las experiencias se hizo en bases de datos especializadas, las cuales concentran artículos científicos que han sido objeto de un proceso riguroso de evaluación para su publicación. Específicamente, la búsqueda se hizo en las bases de Web of Sicence, Scopus y Scielo a partir de la combinación de las siguientes palabras clave: 1) segregación escolar, 2) 
educación no formal, 3) alternativas educativas y 4) América Latina, en inglés y en español. Asimismo, el enfoque de la investigación debía ser el de experiencias dirigidas a niñas, niños y jóvenes. De los resultados de la búsqueda, se eligieron solamente artículos científicos publicados en los últimos seis años que cumplieran con los criterios mencionados. Así, se eligieron cinco artículos a partir de los cuales se pudo hacer un análisis de contenido de diversas experiencias. Si bien cada caso corresponde a la explicación de un contexto particular, cabe destacar que su elección se realizó por su relevancia y por las estrategias de educación no formal propuestas para resolver el problema de segregación educativa y social. Por lo tanto, no representan una generalidad del país en el que se desarrolla la experiencia, sino que destacan su importancia, por las alternativas a la exclusión que se produce en cada entorno. Cabe señalar también que todas las experiencias comparten problemas semejantes en el contexto de segregación, los cuales corresponden a los de la realidad latinoamericana.

\section{Identificación de las experiencias educativas}

Los casos seleccionados se reconocen a partir de cuatro aspectos, fundamentalmente: 1) contexto de segregación, 2) propósitos, 3) características y 4) estrategias en la educación no formal.

\section{Identificación de categorías de análisis}

La revisión de la literatura dio lugar a cuatro categorías teóricas, a partir de las cuales se sintetizó la información recopilada de cada uno de los casos descritos. En el análisis de contenido, se generaron cinco categorías emergentes, las cuales presentan los aportes en la educación no formal que realiza cada una de estas experiencias para contrarrestar el problema de la segregación escolar.

\section{Resultados}

Las experiencias recopiladas destacan aspectos de la educación no formal cuya finalidad es resolver las consecuencias de la segregación escolar.
Los resultados se presentan de acuerdo con las categorías derivadas del análisis realizado: 1) segregación: injusticia e inequidad; 2) educación inclusiva e intercultural; 3) reivindicación de derechos y empoderamiento; 4) pensamiento crítico y diálogo; y 5) educación comunitaria.

\section{Segregación: injusticia e inequidad}

Los casos analizados demuestran la relación entre la segregación escolar y la desigualdad social que caracteriza a la región latinoamericana. Independientemente de las particularidades contextuales de cada país, las propuestas aluden a prácticas que compensan la falta de atención escolar integral en entornos de alta marginación social en América Latina (Krüger, 2019; Villalta y Assael, 2018). Específicamente, las experiencias $A$ y $E$ hacen referencia a la incapacidad de los sistemas escolares y recursos educativos que, a pesar de ser señalados como alternativos, no responden a las necesidades y características del alumnado de determinada condición cultural, económica y social, por lo que se perpetúa en el ámbito escolar el problema de la segregación. La primera experiencia, aunque contaba con medios audiovisuales para incentivar los procesos educativos de los sectores más marginados, no tenía una propuesta dirigida al desarrollo de competencias como el pensamiento crítico, el empoderamiento y la creación de medios propios que contribuyeran al bienestar individual y colectivo, aspectos indispensables para la participación en la defensa y el ejercicio de los derechos ciudadanos (Niederberger, 2012). Del mismo modo, la experiencia $E$ se trata de un caso que cuestiona un proyecto universitario que no atiende a la diversidad cultural del alumnado, por lo que reproduce la exclusión social en el contexto educativo. En este sentido, parte de la metodología en ambas propuestas contempla el análisis de las intervenciones educativas llevadas a cabo hasta el momento para revisar el alcance de las metas educativas deseadas (Florentino, 2014), y la no repetición de la problemática de discriminación establecida en la estructura social (Cabrero y Valencia, 2019). 
Tabla 1. Casos de análisis seleccionados

\begin{tabular}{|c|c|c|c|c|}
\hline & $\begin{array}{l}\text { Contexto de } \\
\text { segregación }\end{array}$ & Propósitos & Caracteristicas & $\begin{array}{c}\text { Estrategias en la educación no } \\
\text { formal }\end{array}$ \\
\hline $\begin{array}{l}\text { Experiencia A } \\
\text { Alfabetización } \\
\text { mediática a través de } \\
\text { Organizaciones No } \\
\text { Gubernamentales } \\
\text { (ONG) (Brasil). }\end{array}$ & $\begin{array}{l}\text { Zona con altos } \\
\text { índices de pobreza } \\
\text { y sectores en riesgo } \\
\text { de exclusión social. }\end{array}$ & $\begin{array}{l}\text { Utilizar medios } \\
\text { de comunicación } \\
\text { alternativos como } \\
\text { vía para promover } \\
\text { el desarrollo de } \\
\text { competencias } \\
\text { fundamentales } \\
\text { para el ejercicio } \\
\text { pleno del acceso a la } \\
\text { información y a la } \\
\text { educación. }\end{array}$ & $\begin{array}{l}\text { Enfoque hacia la infancia y } \\
\text { la juventud. } \\
\text { Contenidos de aprendizaje } \\
\text { centrados en temas } \\
\text { sociales y de reivindicación } \\
\text { de derechos. } \\
\text { Trabajo con profesores y } \\
\text { estudiantes de escuelas } \\
\text { públicas. } \\
\text { Colaboración con } \\
\text { fundaciones, otras ONG e } \\
\text { instituciones públicas. }\end{array}$ & $\begin{array}{l}\text { Acceso a y uso de medios de } \\
\text { comunicación alternativos } \\
\text { para producción de contenidos } \\
\text { propios. } \\
\text { Desarrollo de habilidades } \\
\text { comunicativas con } \\
\text { comprensión crítica de los } \\
\text { contenidos (pensamiento } \\
\text { crítico). } \\
\text { Trabajo por objetivos } \\
\text { integrados para el } \\
\text { empoderamiento mediante la } \\
\text { alfabetización mediática. } \\
\text { Creación de canales de } \\
\text { comunicación permanente con } \\
\text { la comunidad. }\end{array}$ \\
\hline $\begin{array}{l}\text { Experiencia B } \\
\text { Alternativas } \\
\text { de educación } \\
\text { intercultural } \\
\text { basadas en la } \\
\text { sustentabilidad } \\
\text { (México). }\end{array}$ & $\begin{array}{l}\text { Desigualdad social, } \\
\text { laboral, económica } \\
\text { y educativa. } \\
\text { Falta de } \\
\text { oportunidades } \\
\text { laborales en la } \\
\text { comunidad: causa } \\
\text { de migración. }\end{array}$ & $\begin{array}{l}\text { Recuperar y revalorar } \\
\text { los discursos y } \\
\text { prácticas culturales } \\
\text { e identitarias. }\end{array}$ & $\begin{array}{l}\text { Enfoque participativo } \\
\text { de las y los jóvenes en } \\
\text { la recuperación de los } \\
\text { saberes comunitarios } \\
\text { y la transformación del } \\
\text { entorno de desigualdad. } \\
\text { Trabajo con agentes } \\
\text { externos para su } \\
\text { adaptación a las } \\
\text { dinámicas y perspectivas } \\
\text { comunitarias. } \\
\text { Reflexión colectiva } \\
\text { permanente sobre los } \\
\text { conocimientos de la } \\
\text { comunidad que han } \\
\text { permanecido subalternos. } \\
\text { Fortalecimiento de } \\
\text { las redes locales en la } \\
\text { documentación del } \\
\text { conocimiento. }\end{array}$ & $\begin{array}{l}\text { Creación de una comunidad de } \\
\text { aprendizaje fuera del contexto } \\
\text { escolar. } \\
\text { Creación de material } \\
\text { audiovisual como herramienta } \\
\text { educativa para recuperar los } \\
\text { saberes comunitarios. } \\
\text { Producción audiovisual } \\
\text { en talleres participativos y } \\
\text { relacionados con el entorno. } \\
\text { Definición de los parámetros } \\
\text { de ejecución del proyecto por } \\
\text { parte de las y los jóvenes. } \\
\text { Uso del diálogo para la } \\
\text { concientización sobre la } \\
\text { jerarquía de saberes en el } \\
\text { mundo occidental. } \\
\text { Vínculo con agentes clave del } \\
\text { entorno para la creación de } \\
\text { contenidos. }\end{array}$ \\
\hline
\end{tabular}




\begin{tabular}{|c|c|c|c|c|}
\hline & $\begin{array}{l}\text { Contexto de } \\
\text { segregación }\end{array}$ & Propósitos & Características & $\begin{array}{c}\text { Estrategias en la educación no } \\
\text { formal }\end{array}$ \\
\hline $\begin{array}{l}\text { Experiencia C } \\
\text { Miradas a las } \\
\text { alternativas } \\
\text { educativas al fracaso } \\
\text { escolar (Argentina). }\end{array}$ & $\begin{array}{l}\text { Niñas y niños } \\
\text { con repetidas } \\
\text { experiencias de } \\
\text { fracaso escolar en } \\
\text { educación básica. } \\
\text { Contexto de alta } \\
\text { vulnerabilidad } \\
\text { social y económica. } \\
\text { Problemas sociales } \\
\text { asociados a } \\
\text { abandono familiar, } \\
\text { delincuencia y } \\
\text { adicciones de } \\
\text { familiares cercanos. }\end{array}$ & $\begin{array}{l}\text { Nivelar los } \\
\text { aprendizajes } \\
\text { escolares y } \\
\text { fomentar el gusto } \\
\text { por aprender en la } \\
\text { escuela. } \\
\text { Apoyar la } \\
\text { escolarización } \\
\text { exitosa del } \\
\text { alumnado. }\end{array}$ & $\begin{array}{l}\text { Desarrollo de confianza y } \\
\text { autonomía. } \\
\text { Vínculo entre la escuela y el } \\
\text { centro comunitario. } \\
\text { Compromiso de } \\
\text { autoridades con el } \\
\text { desarrollo de la niñez. } \\
\text { Toma de decisiones } \\
\text { consensuada y gestión } \\
\text { colaborativa. } \\
\text { Establecimiento de } \\
\text { redes con agrupaciones } \\
\text { comunitarias, } \\
\text { organizaciones } \\
\text { gubernamentales y no } \\
\text { gubernamentales. } \\
\text { Trabajo colaborativo para } \\
\text { el acceso a otros servicios, } \\
\text { como las becas escolares, la } \\
\text { salud, la alimentación y la } \\
\text { asesoría legal. }\end{array}$ & $\begin{array}{l}\text { Inserción en los barrios. } \\
\text { Redistribución del tiempo } \\
\text { educativo. } \\
\text { Estructura educativa no } \\
\text { graduada. } \\
\text { Trabajo en grupos pequeños y } \\
\text { atención individual. } \\
\text { Participación en actividades } \\
\text { artísticas, deportivas y } \\
\text { proyectos integrados. } \\
\text { Apropiación del espacio } \\
\text { educativo enseñando y } \\
\text { aprendiendo de diversas } \\
\text { maneras. } \\
\text { Concientización de las familias } \\
\text { sobre la importancia de la } \\
\text { escolarización. }\end{array}$ \\
\hline $\begin{array}{l}\text { Experiencia D } \\
\text { Aprendizaje con } \\
\text { niños neorrurales } \\
\text { mayas (México). }\end{array}$ & $\begin{array}{l}\text { Altos índices } \\
\text { de pobreza, } \\
\text { marginación, } \\
\text { repetición y } \\
\text { deserción escolar } \\
\text { de niñas y niños } \\
\text { mayas. } \\
\text { Discriminación } \\
\text { escolar por ser } \\
\text { indígenas. } \\
\text { Fenómeno de } \\
\text { migración en busca } \\
\text { de trabajo. }\end{array}$ & $\begin{array}{l}\text { Fortalecer el } \\
\text { desarrollo integral } \\
\text { de las niñas y } \\
\text { los niños de la } \\
\text { comunidad. } \\
\text { Proponer } \\
\text { alternativas } \\
\text { educativas flexibles. } \\
\text { Desarrollar } \\
\text { aprendizajes más } \\
\text { allá del currículo } \\
\text { escolar. }\end{array}$ & $\begin{array}{l}\text { Colectivo constituido por } \\
\text { agentes comunitarios } \\
\text { y otros fuera de la } \\
\text { comunidad. } \\
\text { Organización de } \\
\text { actividades por profesores } \\
\text { y alumnado de la } \\
\text { Universidad, personas de } \\
\text { la ciudad y de la propia } \\
\text { comunidad. } \\
\text { Participación horizontal en } \\
\text { las actividades educativas. } \\
\text { Reconocimiento y respeto } \\
\text { hacia niñas y niños, y su } \\
\text { diversidad. }\end{array}$ & $\begin{array}{l}\text { Talleres de derechos humanos, } \\
\text { educación para la paz, } \\
\text { artesanía, idiomas, pueblos } \\
\text { originarios, entre otros. } \\
\text { Visitas arqueológicas. } \\
\text { Actividades deportivas y } \\
\text { artísticas. } \\
\text { Apoyo psicopedagógico a niñas } \\
\text { y niños. } \\
\text { Creación de espacios de } \\
\text { convivencia y aprendizaje para } \\
\text { todos. }\end{array}$ \\
\hline $\begin{array}{l}\text { Experiencia E } \\
\text { Universidad } \\
\text { Intercultural de } \\
\text { los Pueblos del Sur } \\
\text { (México). }\end{array}$ & $\begin{array}{l}\text { Analfabetismo, } \\
\text { absentismo escolar } \\
\text { y rezago educativo } \\
\text { de jóvenes } \\
\text { indígenas rurales. } \\
\text { Falta de adaptación } \\
\text { de la universidad } \\
\text { tradicional a } \\
\text { los saberes } \\
\text { comunitarios. } \\
\text { Falta de oferta } \\
\text { educativa y } \\
\text { empleo: causas } \\
\text { de migración a la } \\
\text { ciudad. }\end{array}$ & $\begin{array}{l}\text { Buscar modos } \\
\text { de aprendizaje } \\
\text { alternativos a } \\
\text { la universidad } \\
\text { intercultural estatal. } \\
\text { Propiciar el } \\
\text { desarrollo individual } \\
\text { y social de los } \\
\text { pueblos indígenas. } \\
\text { Brindar un proceso } \\
\text { educativo vinculado } \\
\text { al contexto } \\
\text { comunitario. }\end{array}$ & $\begin{array}{l}\text { Generación de aprendizajes } \\
\text { colectivos entre } \\
\text { estudiantes, profesores } \\
\text { de la Universidad } \\
\text { Intercultural y miembros } \\
\text { de la comunidad. } \\
\text { Proyecto educativo creado } \\
\text { por la comunidad indígena. } \\
\text { Reconocimiento del } \\
\text { derecho indígena al } \\
\text { bienestar económico y } \\
\text { social. } \\
\text { Educación gratuita para } \\
\text { el alumnado, financiada } \\
\text { por la comunidad y } \\
\text { organizaciones externas. }\end{array}$ & $\begin{array}{l}\text { Metodología que fortalece el } \\
\text { pensamiento lógico, reflexivo } \\
\text { y crítico. } \\
\text { Desarrollo de proyectos } \\
\text { productivos que fortalezcan a } \\
\text { la comunidad. } \\
\text { Impulsar los saberes } \\
\text { tradicionales. } \\
\text { Planes educativos flexibles y } \\
\text { adaptables al contexto cultural } \\
\text { y las condiciones de vida. } \\
\text { Planta docente formada en } \\
\text { competencias interculturales } \\
\text { proveniente de diferentes } \\
\text { universidades. }\end{array}$ \\
\hline
\end{tabular}

Fuente: elaboración propia a partir de Durantini (2018); Mijangos et al. (2017); Pegurer y Martínez (2016); Sandoval y Mendoza (2017); Alonso et al. (2014). 
Tabla 2. Proceso de categorización

\begin{tabular}{|c|c|c|}
\hline Categorias teóricas & Componentes de las experiencias educativas & Categorias emergentes \\
\hline $\begin{array}{l}\text { Dimensiones de la } \\
\text { segregación educativa } \\
\text { y social. }\end{array}$ & $\begin{array}{l}\text { Contexto de pobreza y vulnerabilidad a la exclusión social. } \\
\text { Exclusión y discriminación en las estructuras educativas tradicionales. } \\
\text { Falta de oportunidades laborales, educativas y de desarrollo social. } \\
\text { Pérdida de conocimientos que forman parte de la identidad cultural. } \\
\text { Experiencias de fracaso escolar, rezago educativo y falta de desarrollo de } \\
\text { competencias básicas para la vida. } \\
\text { Problemas sociales asociados a la violencia, la delincuencia o las adicciones. }\end{array}$ & $\begin{array}{l}\text { Segregación: injusticia } \\
\text { social e inequidad. }\end{array}$ \\
\hline $\begin{array}{l}\text { Vínculo entre la } \\
\text { educación formal y no } \\
\text { formal. }\end{array}$ & $\begin{array}{l}\text { Reconocimiento de las características culturales y sociales del alumnado. } \\
\text { Alternativas en las estrategias de enseñanza y aprendizaje. } \\
\text { Organización educativa flexible. } \\
\text { Atención a las necesidades individuales de aprendizaje. } \\
\text { Planes educativos adaptables a las características y condiciones del } \\
\text { alumnado. } \\
\text { Formación del profesorado en competencias interculturales. }\end{array}$ & $\begin{array}{l}\text { Educación inclusiva e } \\
\text { intercultural. }\end{array}$ \\
\hline $\begin{array}{c}\text { Formación para la } \\
\text { ciudadanía. }\end{array}$ & $\begin{array}{l}\text { Producción de contenidos propios. } \\
\text { Derechos de acceso a la información y a la educación. } \\
\text { Participación para la organización autogestionaria. } \\
\text { Implicación en las tomas de decisiones. } \\
\text { Proyectos integrados con actividades artísticas, deportivas y comunitarias. } \\
\text { Horizontalidad en la organización de las actividades educativas. }\end{array}$ & $\begin{array}{l}\text { Reivindicación de } \\
\text { derechos, autonomía y } \\
\text { empoderamiento. } \\
\text { Pensamiento crítico y } \\
\text { diálogo. }\end{array}$ \\
\hline $\begin{array}{l}\text { Trabajo integrado y } \\
\text { colaborativo. }\end{array}$ & $\begin{array}{l}\text { Vínculo permanente entre los procesos educativos y comunitarios. } \\
\text { Proyectos enfocados a la transformación de la comunidad. } \\
\text { Perspectiva comunitaria en el trabajo integrado. } \\
\text { Fortalecimiento de los lazos comunitarios y aprendizajes colectivos. } \\
\text { Financiamiento compartido. } \\
\text { Colaboración entre agentes comunitarios y otros agentes. } \\
\text { Compromiso con el desarrollo colectivo y reflexión permanente. } \\
\text { Establecimiento de redes entre organizaciones y gestión colaborativa. }\end{array}$ & Educación comunitaria. \\
\hline
\end{tabular}

Fuente: Elaboración propia. 
Como parte de la problemática de exclusión social en la que se contextualiza el fenómeno de la segregación escolar, además de las cuestiones relacionadas con la discriminación en los espacios escolares, las experiencias $B$ y $D$ enfatizan en la falta de oportunidades laborales y educativas en las comunidades de origen del alumnado más vulnerable, cuya consecuencia es la migración y su característica la incapacidad del sistema escolar para adaptarse a sus necesidades y condiciones socioeconómicas (Bustos y Garín, 2017; Velasco y Rentería, 2019). Como consecuencia, los sectores en mayor desigualdad social presentan altos índices de deserción y rezago educativo, así como dificultades para el desarrollo de las competencias básicas para la vida. De este modo, estas experiencias confirman la relación entre la segregación escolar y la precarización de la vida (Krüger, 2019), donde los sectores socioeconómicamente más vulnerables de la región son los que se encuentran más excluidos dentro del sistema educativo.

En relación con lo anterior, las experiencias $C, D$ y $E$ muestran la desigualdad educativa en la población indígena y en los sectores que se enfrentan a la violencia social. Al respecto, los casos $D$ y $E$ aluden al vínculo entre el origen étnico-cultural con la falta de oportunidades educativas de calidad que posibiliten el desarrollo de las competencias escolares básicas (Vázquez, 2016). Asimismo, la experiencia C demuestra la problemática a la que se enfrenta la niñez y la juventud latinoamericana para acceder a opciones formativas que favorezcan su desarrollo en entornos de alta complejidad social, hecho que afecta sus procesos de aprendizaje y su integridad física y emocional (Trucco e Inostroza, 2017). De esta manera, estos casos expresan la segregación escolar como un fenómeno vinculado a las limitaciones del sistema educativo para atender la diversidad cultural y lingüística del alumnado, así como sus condiciones sociales y económicas (Murillo y Martínez, 2017; Murillo, 2016; Vázquez, 2016). Esto conforma una estructura escolar profundamente desigual, donde la organización social condiciona el logro de los aprendizajes básicos que propician tanto el bienestar individual como la construcción de una sociedad más justa en la que todas las personas tengan las mismas oportunidades educativas y posibilidades en el logro de todos los aprendizajes deseados. Por lo tanto, las experiencias analizadas representan una alternativa a una educación formal que conlleva el problema de la segregación como una de sus características centrales (Murillo, 2016).

\section{Educación inclusiva e intercultural}

Además de la necesidad de acceso a las mismas oportunidades educativas, la perspectiva inclusiva e intercultural es la referencia en la que se enmarca la mayor parte de las alternativas educativas analizadas, como un enfoque que reconoce que no es suficiente la integración del alumnado al sistema escolar, pues se requiere valorar su diversidad cultural en cuanto a sus condiciones de educabilidad, considerando que se trata de los sectores que se encuentran en mayor desventaja social dentro las sociedades en las que se desenvuelven (Hurtado et al., 2019). Concretamente, los casos $B$ y $E$ se dirigen a la recuperación de los saberes del alumnado indígena, con la finalidad de revalorar su identidad y sus prácticas culturales-comunitarias para fortalecer su desarrollo individual y contribuir al de su colectivo. De esta manera, en la educación no formal se crean espacios de participación en donde el alumnado toma decisiones sobre la incorporación de los contenidos que son parte del proyecto audiovisual para la recuperación de los saberes comunitarios (caso $B$ ) y participa en actividades de aprendizaje relacionadas con el conocimiento de los derechos humanos, el arte y el deporte, y de la propia cultura (caso E). Las acciones educativas de ambos casos representan una alternativa a la educación escolar que limita el aprendizaje de los grupos que no son parte de la cultura hegemónica. Por lo tanto, la educación no formal contribuye al logro de aprendizajes fuera del currículo formal, pero de gran relevancia para el desarrollo personal y la cohesión social (Chávez, 2013; Ortega, 2005). 
Por su parte, en las experiencias C y D se explican estrategias que rompen la estructura tradicional del sistema escolar para fortalecer las competencias básicas mediante una estructura educativa orientada a la atención de las diferentes formas de aprendizaje. En el caso $C$, se replanteó la organización de las actividades de aprendizaje, centrándose en un establecimiento horario acorde a las formas de vida del alumnado, la eliminación de la graduación académica por edad, así como la atención personalizada de las necesidades de aprendizaje. De esta manera, la propuesta plantea el logro de objetivos educativos mediante la identificación de las diferentes circunstancias que limitan el aprendizaje dentro del ámbito formal de la educación, por lo que es fundamental el reconocimiento de las características personales y culturales, así como de las condiciones de vida de los estudiantes (De la Cruz, 2017). Aunque uno de los objetivos de esta experiencia es incentivar una efectiva integración del alumnado en el sistema escolar e incrementar su interés por los aprendizajes académicos correspondientes, los esfuerzos también se dirigen hacia el logro de competencias complejas asociadas al desarrollo en diversas áreas, tanto personales como sociales, destacando la importancia de la educación como factor de inclusión social (Ramírez, 2014).

Igualmente, en la experiencia $D$ se brinda una atención psicopedagógica individualizada y se crean espacios de convivencia y aprendizaje, en los cuales se amplía el conocimiento académico, reforzando los saberes del alumnado. En este sentido, el proyecto se enfoca a incentivar el desarrollo integral de las capacidades tomando en cuenta las características del alumnado y fomentando valores como el respeto y la tolerancia (Rubaii, 2016). En ambos casos, la finalidad es estructurar procesos educativos ajustados a las necesidades de los estudiantes y a sus características culturales. Estas experiencias demuestran que el perfil del alumnado, que suele ser segregado dentro del sistema escolar, tiene la posibilidad de ser reconocido a través de estrategias que desarrollen sus capacidades y que se basen en una organización que se ajusta a sus requerimientos y a su contexto. Dichas estrategias diversifican las formas de enseñanza y establecen un entorno de aprendizaje en el que niñas y niños se sienten reconocidos, aspectos característicos de la inclusión desde la educación (Ramírez, 2016).

Las alternativas educativas en el ámbito no formal revaloran los conocimientos de los estudiantes, así como sus referentes culturales y comunitarios, contando con una enseñanza que se enfoca en atender a la diversidad. Esto se visualiza específicamente en los casos $B$ y E. En el primero, esta revalorización de conocimientos se realiza a través de la participación de los jóvenes en el diseño y práctica del proyecto comunitario en el que se involucra a diversos agentes del entorno. De esta manera, el aprendizaje deriva de una reconstrucción propia de los saberes colectivos y refuerza los saberes que dan identidad a su historia personal, familiar y comunitaria. En este sentido, el proyecto ha permitido recuperar los conocimientos que son excluidos del currículo escolar y reconoce la necesidad de integrar los saberes históricamente marginados dentro de los procesos de enseñanza-aprendizaje (Vargas, 2019). Asimismo, en la experiencia $E$ la flexibilidad de los planes educativos, la implementación de prácticas en las que el alumnado puede tomar decisiones sobre los aspectos que impactan en su formación y en su entorno, así como la preparación docente dentro del marco de la interculturalidad, y su diversidad disciplinaria y científica, favorecieron los aprendizajes del alumnado partiendo de sus conocimientos y prácticas culturales, generando procesos educativos con dinámicas en las que todas las personas se sienten incluidas.

\section{Reivindicación de derechos, autonomía y empoderamiento}

Aunada a la categoría anterior, las estrategias de la educación no formal buscan fortalecer el ejercicio de los derechos ciudadanos, mediante el protagonismo del alumnado en los proyectos y acciones 
educativas. De esta manera, la participación es un componente central (Ramírez, 2016) en las experiencias analizadas. Se identifica que esta categoría se relaciona con la de educación inclusiva e intercultural en la medida en que, al centrar a los estudiantes como el eje de los procesos educativos, se reconocen sus referentes culturales y sus características de aprendizaje. Estos aspectos se evidencian en el caso $E$, donde el liderazgo de la comunidad indígena permitió la creación del proyecto educativo y donde los jóvenes elaboraron iniciativas que contribuyeran al desarrollo de su comunidad, forjando un aprendizaje apegado a su contexto. De este modo, la participación como eje esencial de las prácticas hizo que, en el ámbito de la educación no formal, se contribuyera al fortalecimiento de la cultura democrática y de las capacidades que esta conlleva (Silva et al., 2018).

Al respecto, en las experiencias $B$ y $D$ la participación de niñas, niños y jóvenes en los procesos educativos se desarrolló con base en un principio de igualdad de oportunidades en la toma de decisiones en relación con las acciones que se llevaron a cabo. Así, los jóvenes fueron quienes determinaron los lineamientos para la puesta en práctica del proyecto a través del diálogo y la autoorganización (caso $B$ ), fomentando dinámicas colaborativas con las aportaciones de cada uno de los agentes intervinientes en el proyecto, basados en sus propias capacidades de aportación (caso D). En este sentido, se destaca, por un lado, la relevancia del intercambio de saberes, el conocimiento mutuo y la participación como formas de generar sentido de pertenencia al trabajo que se realiza, y, por otro, la importancia de un trabajo integrado en el que participan diferentes actores que favorecen la educación (Subirats y Albaigés, 2006). Por lo tanto, se quiebra la relación jerárquica que caracteriza a la educación escolar y que invisibiliza el poder del alumnado para decidir sobre el transcurso de su propio proceso de aprendizaje.

Otras de las estrategias de participación se destacan en las experiencias $A$ y $C$, las cuales hacen referencia a la integración de las acciones de los estudiantes en proyectos que involucran diversas áreas del conocimiento. En el caso $A$, la participación de la población en la generación de contenido audiovisual a partir de su conocimiento del entorno fomentó la apropiación de los medios de producción de información y comunicación, relacionando el contenido con aquellos sucesos del entorno que son de su interés y que requieren problematizarse usando los cuestionamientos y búsqueda de soluciones por parte de quienes tienen un vínculo directo con el contexto. Asimismo, en el caso C, la participación de niñas y niños como base de las acciones del proyecto fortaleció su confianza y autonomía, mismas que se limitaban en las dinámicas de la educación escolar. De esta manera, la educación no formal rebasa una instrucción compensatoria de los aprendizajes escolares y transforma la problemática de segregación en oportunidades para desarrollar capacidades necesarias en la toma de decisiones individuales y sociales (Cabrero y Valencia, 2019). En consecuencia, se fortalece una visión educativa en la que los sectores más vulnerables tienen injerencia en los procesos que impactan en su vida y que determinan sus posibilidades de ejercer sus derechos.

Las experiencias analizadas constituyen procesos de formación en los que se fomenta la colaboración y el desarrollo de competencias como la independencia en la valoración de sus propias tareas. Al ser los estudiantes los principales actores del proceso educativo tienen más probabilidades de acceder a los recursos que necesitan y que no siempre están disponibles en la organización del sistema escolar. Sobre este punto, en el caso $A$ la información es un recurso al que los participantes pueden acceder fácilmente y con el que pueden trabajar, desarrollando sus capacidades de autonomía y toma de decisiones.

\section{Pensamiento crítico y diálogo}

El logro de capacidades como la autonomía y el empoderamiento requiere de habilidades asociadas al pensamiento crítico y al diálogo. Sobre este punto, las experiencias $A, B$ y $E$ se enfocan en la construcción 
de proyectos colaborativos donde la comunicación y el uso de las capacidades analíticas y reflexivas para la construcción de contenidos propios son fundamentales. En el caso $A$, se impulsó un trabajo cooperativo entre el profesorado y el alumnado de diversas escuelas públicas, con la finalidad de generar contenidos acordes con el entorno y en los que todas las personas se sintieran identificadas con la información que se compartía, haciendo uso de distintos medios de comunicación. Asimismo, en la experiencia $B$ la organización autónoma de los jóvenes fue la principal estrategia para la puesta en práctica del proyecto, a través de la cual se generó contenido audiovisual ligado a su contexto y con los referentes culturales de su comunidad, lo que fortaleció su sentido de pertenencia e identidad en relación con los otros contextos en los que se desenvuelven. De manera similar, en el caso $E$ las dinámicas de participación posibilitaron que el proyecto educativo fuera apropiado por el alumnado, lo cual propició que reconocieran sus derechos para tomar decisiones en torno a los aspectos que afectan su desarrollo. La autoorganización, el trabajo colaborativo y la participación sistemática en la coordinación y puesta en práctica de las diversas acciones propuestas se lograron mediante el diálogo y el análisis continuo de la realidad, aspectos fundamentales en el desarrollo individual y colectivo de las poblaciones más segregadas. Así, se evidencia que la transformación de una realidad de marginación educativa y social requiere el fortalecimiento de las capacidades críticas, e intercambio y cooperación para la construcción de una ciudadanía incluyente (Unesco, 2015).

Por lo tanto, las elecciones que paulatinamente se realizaron en estas iniciativas estuvieron sustentadas en un cuestionamiento continuo, así como en la puesta en común de diferentes perspectivas entre sus integrantes. Esto, a su vez, les permitió valorar los saberes que conforman su identidad y distinguir los referentes externos, haciendo un ejercicio de retroalimentación de los conocimientos propios y enriqueciendo continuamente los aprendizajes. En este sentido, el ejercicio de las capacidades de diálogo y de pensamiento crítico ha posibilitado la identificación de la desigualdad en la que se encuentran los conocimientos comunitarios en relación con los hegemónicos, así como las situaciones de exclusión en la que se han encontrado dentro del ámbito formal de la educación (Martínez, 2016; Walsh, 2014). De este modo, la reflexión, el cuestionamiento y el análisis, así como la comunicación participativa y colaborativa, son capacidades centrales en los procesos educativos, que se dirigen a cuestionar las estructuras que originan la desigualdad y la exclusión, con la finalidad de propiciar procesos educativos que incentiven el desarrollo de todas las personas que forman parte de estos.

De esta manera, las acciones implicadas en los proyectos analizados contribuyeron al desarrollo integral fundado en la reciprocidad de las aportaciones de todos y la continua evaluación de las diversas perspectivas en una integración de educación colectiva. Así, se destaca que las acciones de cooperación implican la transversalidad de los aportes de los involucrados en el proceso de educación (Opertti, 2015), lo cual, además de lo anteriormente expuesto, retroalimenta los aprendizajes escolares (Ortega, 2005) y contribuye a la inclusión del alumnado más vulnerable.

\section{Educación comunitaria}

Las categorías descritas dan lugar a otro de los aspectos fundamentales de las alternativas de educación analizadas: la educación comunitaria, la cual se asocia a la participación y al vínculo entre los diferentes agentes sociales en los procesos educativos, además de los que trabajan en el ámbito escolar (Ferrer et al., 2006), con base en un principio de responsabilidad compartida (Sabariego, 2009).

La visión comunitaria de la educación aparece de diferente manera en cada una de las experiencias. En el caso $A$ el diálogo permanente entre los distintos participantes del proyecto es un elemento 
que permite que los grupos que forman parte de él establezcan lazos de comunicación para el análisis y el establecimiento de acuerdos sobre los contenidos a integrar. Así se conforma un conjunto de ideas sustentadas en el compromiso de todos los involucrados, especialmente la infancia y la juventud a la que se dirigen las acciones educativas. Por su parte, en la experiencia $B$ la perspectiva comunitaria constituyó la base para que los agentes externos adaptaran sus acciones a los requerimientos de la comunidad. Mediante estrategias educativas que privilegiaron el diálogo y la reflexión continua sobre las diversas acciones emprendidas, se creó una comunidad de aprendizaje fuera del entorno escolar, fomentando dinámicas de ayuda mutua y solidaridad, lo que posibilitó diversas identificaciones entre los participantes y llevó a establecer lazos para el bienestar colectivo fundado en las demandas sociales (Ramírez, 2017). Por consiguiente, la educación no formal coadyuvó en la transformación de la segregación con la creación de vínculos que incorporaron las visiones, necesidades y aportaciones de todas las personas y disminuyeron las dificultades que impedían el logro de los aprendizajes.

Complementario a lo anterior, el ámbito no formal de la educación generó vínculos con las acciones escolares. Así, en las experiencias C y E el trabajo de los agentes comunitarios y la colaboración entre los distintos involucrados buscaron un nexo con las actividades que se llevaban a cabo en la escuela, de tal manera que los procesos de educación no formal tuvieron entre sus propósitos retroalimentar los aprendizajes del sistema escolar. De esta manera, en todas las experiencias, el desarrollo de los aprendizajes requirió un trabajo que integrara las prácticas de los distintos actores implicados en los procesos de educación (Ramírez, 2014). Sin embargo, es un tipo de colaboración orientada por las necesidades y la perspectiva de la comunidad, es decir, de adentro hacia afuera, por lo que los agentes externos a la misma debieron adecuar sus acciones a las demandas de los integrantes de la propia comunidad.

\section{Discusión y conclusiones}

La segregación escolar, como expresión de la exclusión social, se manifiesta en todas las experiencias analizadas a través del analfabetismo, la repetición, el rezago educativo y la deserción, indicadores de la multidimensionalidad de la segregación (Murillo y Martínez, 2019; Murillo et al., 2018). Por un lado, se visualiza que las alternativas de la educación formal para las comunidades indígenas han resultado en una educación que no ha correspondido a sus necesidades y características culturales y lingüísticas, pero sí ha brindado una escolarización de menos calidad a un sector que ha sido homogeneizado y segregado, al no adaptarse a las exigencias del sistema. Asimismo, se observa la dimensión de uniformidad, al no ofrecer el apoyo pertinente para que el alumnado con mayor exclusión social pudiera desarrollar los aprendizajes esperados dentro de la estructura formal de educación.

Por otra parte, este análisis permite evidenciar cómo los procesos educativos alternativos, en el ámbito no formal, contribuyen a la inclusión educativa y, por lo tanto, a resolver las consecuencias de la segregación escolar. En este sentido, las estrategias orientadas a la inclusión traen una transformación de las dinámicas de exclusión que genera el sistema escolar, confirmando este proceso como el componente opuesto de la segregación (Murillo y Duk, 2016). A diferencia de aquellas acciones que fomentan la inclusión dentro de la estructura escolar, la educación no formal plantea la posibilidad de crear dinámicas disruptivas del sistema escolar desde afuera. Debido a la inflexibilidad del propio sistema, la educación no formal consigue retroalimentar los aprendizajes que no logra la escuela, pero a través de otros mecanismos, como la participación y la convivencia de la diversidad (Cabrero y Valencia, 2019), los cuales son limitados en la estructura tradicional de la educación formal. Al respecto, las experiencias analizadas alcanzaron a desarrollar competencias no solamente para que el alumnado continuara sus trayectorias académicas, sino tam- 
bién para mejorar su desenvolvimiento dentro de su entorno. De esta manera, las alternativas educativas en este ámbito ofrecen una perspectiva transformadora de la realidad de exclusión educativa y social.

Además, el trabajo en la educación no formal fortalece la formación para la ciudadanía, al desarrollar competencias relacionadas con la cooperación, el trabajo integrado, la participación y el pensamiento crítico, aspectos que contribuyen a la cohesión social (Chávez, 2013) y, por lo tanto, son necesarios para contrarrestar un sistema que tiende a la segregación. Si bien estas habilidades forman parte de los planes de la educación escolar, el problema de la exclusión limita una formación para todos en este sentido. Por lo tanto, los múltiples vínculos que se pueden establecer en el ámbito no formal de la educación constituyen una oportunidad para generar otras posibilidades de formación fuera de una estructura que insiste en excluir históricamente a determinados sectores.

Por último, cabe señalar que en la realización de este estudio se reafirmó la falta de investigaciones sobre segregación escolar en el contexto de América Latina (Murillo, 2016), específicamente estudios que presenten propuestas alternativas que permitan visualizar estrategias para resolver las distintas dinámicas que continúan reproduciendo esta problemática. A pesar de que la educación no formal se ha delimitado como un ámbito que propicia la generación de procesos educativos innovadores, es escasa la creación de conocimiento que vincule alternativas en esta área para resolver fenómenos como la segregación escolar. Así, este análisis pretende aportar una reflexión en este sentido, con miras a destacar la importancia de este tema en el contexto de desigualdad que se perpetúa en la región latinoamericana.

\section{Referencias}

Alonso, L., Hernández, V. M. y Solís, E. (2014). La Universidad Intercultural de los pueblos del sur. Una opción de educación no formal en el estado de Guerrero, México. Revista Mexicana de Investigación Educativa, 19(60), 103-128. http://www.scielo.org.mx/scielo.php?script=sci_arttext\&pid=S1405-66662014000100006

Ascorra, P., López, V., Núñez, C. G., Bilbao, M. A., Gómez, G.y Morales, M. (2016). Relación entre segregación y convivencia escolar en escuelas públicas chilenas. Universitas Psychologica, 15(1), 65-78. http://dx.doi.org/10.11144/ Javeriana.upsy15-1.rsce

Bustos, R. y Gairín, J. (2017). Adaptación académica de estudiantes migrantes en contextos de frontera. Calidad en la Educación, 46, 193-220. http://dx.doi.org/10.4067/S0718-45652017000100193

Cabrero, J. y Valencia, R. (2019). TIC para la inclusión: una mirada desde Latinoamérica. Aula Abierta, 48(2), 139146. https://doi.org/10.17811/rifie.48.2.2019.139-146

Chávez, J. (2013). Universidad Nacional Agraria La Molina-Perú: inclusión social y discriminación social. Revista Lusófona de Educación, 24, 141-155. https://revistas.ulusofona.pt/index.php/rleducacao/article/view/4181

Córdoba, C., Rojas, K. y Azócar, J. (2016). Selección de alumnos y co-pago como factores de segregación escolar. Presentación de tres casos de estudio. Psicoperspectivas. Individuo y Sociedad, 15(1), 102-116. http://dx.doi. org/10.5027/psicoperspectivas-Vol15-Issue1-fulltext-642 
De la Cruz, G. (2017). Igualdad y equidad en educación: retos para una América Latina en transición. Educación, 26(51), 159-178. https://doi.org/10.18800/educacion.201702.010

Durantini, C. (2018). Miradas a las alternativas educativas al fracaso escolar. Políticas, experiencias, conceptualizaciones y estudio de caso a inicios del S. XXI. Revista de la Escuela de Ciencias de la Educación, 13(2), 121-141. https://doi.org/10.35305/rece.v2i13.387

Echeita, G. (2013). Inclusión y exclusión educativa. De nuevo "voz y quebranto". Revista Iberoamericana sobre Calidad, Eficacia y Cambio en Educación, 11(2), 100-118. https://www.redalyc.org/pdf/551/55160202.pdf

Ferrer, G., Albaigés, B. y Massot, M. (2006). La participació de les famílies en el treball integrat. En J. Subirats y B. Albaigés (eds.), Finestra oberta (pp. 92-110). Fundación Jaume Bofill.

Florentino, B. (2014). Reconstruir la ciudadanía en un entorno de incertidumbre: una tarea educativa. Postconvencionales: Ética, Universidad, Democracia, 7-8, 19-31. http://saber.ucv.ve/ojs/index.php/rev_post/article/ view/6882\#.YAn2g-hKiUk

Guix, J. (2008). El análisis de contenidos: ¿qué nos están diciendo? Revista de Calidad Asistencial, 23(1), 26-30. https://doi.org/10.1016/S1134-282X(o8)70464-0

Hurtado, Y. M., Mendoza, R. S. y Viejó, A. B. (2019). Los desafíos de la formación docente inclusiva: perspectivas desde el contexto latinoamericano. Revista Internacional de Apoyo a la Inclusión, Logopedia, Sociedad y Multiculturalidad, 5(2), 98-110. https://doi.org/10.17561/riai.v5.n2.9

Krüger, N. (2019). La segregación por nivel socioeconómico como dimensión de la exclusión educativa: 15 años de evolución en América Latina. Archivos Analíticos de Politicas Educativas, 27(8), 1-37. http://dx.doi. org/10.14507/epaa.27.3577

López, F. (2002). El análisis de contenido como método de investigación. Revista de Educación, 4, 167-179. http:// rabida.uhu.es/dspace/bitstream/handle/10272/1912/b15150434.pdf

Martínez, C. (2016). Conocimiento occidental y saberes indígenas en la educación intercultural bilingüe en el Ecuador. Alteridad. Revista de Educación, 11(2), 206-220. http//doi.org/10.17163/alt.v11n2.2016.06

Mijangos, J. C., Castillo, C. y Reyes, N. (2017). Aprendizaje con niños neorrurales mayas: una experiencia de educación no formal. Sinéctica. Revista Electrónica de Educación, 49, 1-18. https://sinectica.iteso.mx/index.php/ SINECTICA/article/view/717

Murillo, J. (2016). Midiendo la segregación escolar en América Latina. Un análisis metodológico utilizando el TERCE. Revista Iberoamericana sobre Calidad, Eficacia y Cambio en Educación, 14(4), 33-6o. http://dx.doi. org/10.15366/reice2016.14.4.002

Murillo, J. y Belavi, G. (2018). Segregación escolar de estudiantes inmigrantes pobres en España. Scripta Nova. Revista Electrónica de Geografía y Ciencias Sociales, 22(603), 1-27. https://doi.org/10.1344/sn2018.22.19894 
Murillo, J.y Duk, C. (2016). Segregación escolar e inclusión. Revista Latinoamericana de Educación Inclusiva, 10(2), 11-13. https://doi.org/10.4067/So718-73782016000200001

Murillo, J., Duk, C. y Martínez, C. (2018). Evolución de la segregación socioeconómica de las escuelas en América Latina. Estudios Pedagógicos, 44(1), 157-179. https://doi.org/10.4067/S0718-07052018000100157

Murillo, J. y Martínez, C. (2017). Estimación de la magnitud de la segregación escolar en América Latina. Revista Internacional de Investigación en Educación, 9(19), 11-30. https://doi.org/10.11144/Javeriana.m9-19.emse

Murillo, J. y Martínez, C. (2019). Perfiles de segregación escolar por nivel socioeconómico en España y sus Comunidades Autónomas. Relieve, 25(1), 1-20. http://doi.org/10.7203/relieve.25.1.12917

Nacak, A. y Yücesoy, Y. (2020). Art education for communication in the society: A content analysis. Revista Tempos Espaços en Educaçao, 13(32), 1-12. http://dx.doi.org/10.20952/revtee.v13i32.13110

Niederberger, A. (2012). Reinventar la ciudadanía. Acerca de la conexión entre democracia, derechos y legitimidad en el orden político global. Eidos, 17, 134-167. http://www.scielo.org.co/scielo.php?script=sci_arttext\&p id $=$ S1692-88572012000200006

Olcott, D. (2013). Nuevas líneas de aprendizaje: potenciar el uso de recursos educativos abiertos para reforzar la educación no formal. Revista de Universidad y Sociedad del Conocimiento, 10(1), 151-169. http://dx.doi. org/10.7238/rusc.v10i1.1562

Opertti, R. (2015). La educación inclusiva: perspectiva internacional y desafíos en América Latina. Diálogos Pedagógicos, 13(25), 165-182.

Ortega, J. (2005). La educación a lo largo de la vida: la educación social, la educación escolar, la educación continua... todas son educaciones formales. Revista de Educación, 338, 167-175. https://dialnet.unirioja.es/servlet/articulo?codigo $=1370849$

Ortiz, I. (2015). Escuelas inclusivas en el contexto de segregación social del sistema escolar chileno. Calidad en la Educación, 42, 93-122. http://dx.doi.org/10.4067/S0718-45652015000100004

Pegurer, M. y Martínez, J. F. (2016). Alfabetización mediática en Brasil: experiencias y modelos en educación no formal. Revista Cientifica de Educomunicación, 24(49), 39-78. http://dx.doi.org/10.3916/C49-2016-04

Ramírez, A. (2014). La educación escolar como factor de inclusión social desde un planteamiento sistémico. El caso de la niñez jornalera migrante en los valles de Culiacán, Sinaloa (México) [Tesis de doctorado]. Facultat de Pedagogia, Universitat de Barcelona. http://diposit.ub.edu/dspace/handle/2445/57624

Ramírez, A. (2016). Repensar la inclusión social desde la educación: algunas experiencias en América Latina. Revista Internacional de Educación para la Justicia Social, 5(1), 177-194. https://doi.org/10.15366/riejs2016.5.1

Ramírez, A. (2017). La educación con sentido comunitario: reflexiones en torno a la formación del profesorado. Educación, 26(51), 79-94. https://doi.org/10.18800/educacion.201702.004. 
Rubaii, N. (2016). Promoting social equity, diversity, and inclusion through accreditation. Comparing national and international standards for public affairs programs in Latin America. Quality Assurance in Education, 24(4), 541-561. https://doi.org/10.1108/QAE-02-2016-0007

Sabariego, M. (2009). La educación intercultural como factor de cambio social: modelos de intervención y acción educativa. En M. de Esteban (ed.), El diálogo intercultural en España: un requisito de la educación y cultura de paz (pp. 193-264). Fundación Europea Sociedad y Educación.

Sandoval, J. C. y Mendoza, R. G. (2017). Intercultural educational alternatives based on sustainability from Mexico: beyond school and cultural belonging. Intercultural Education, 4, 373-389. http://dx.doi.org/10.1080/14675 986.2017.1334313

Silva, M., Llaña, M., Maldonado, F. y Baeza, A. (2018). Algunos desafíos curriculares de la formación ciudadana y la diversidad en Chile. Educación, 27(53), 155-173. https://doi.org/10.1880o/educacion.201802.009.

Subirats, J. y Albaigés, B. (2006). Educació i comunitat. Reflexions a l'entorn del treball integrat dels agents educatius. Fundación Jaume Bofill.

Trucco, D. e Inostroza, P. (2017). Las violencias en el espacio escolar. Cepal.

Unesco (2015). Educación para la ciudadanía mundial. Temas y objetivos de aprendizaje. Unesco.

Vargas, P. A. (2019). Estrategias en disputa: educación superior y poblaciones indígenas en la construcción de los Estados-nación latinoamericanos. Revista Cultura y Representaciones Sociales, 13(26), 97-128. https://doi. org/10.28965/2019-26-05

Vázquez, E. (2016). Segregación escolar por nivel socioeconómico: midiendo el fenómeno y explorando sus determinantes. Económica, 42, 121-184. https://www.researchgate.net/publication/238597056_Segregacion_ Escolar_por_Nivel_Socioeconomico_Midiendo_el_Fenomeno_y_Explorando_sus_Determinantes

Velasco, L. y Rentería, D. (2019). Diversidad e interculturalidad: la escuela indígena en contextos de migración. Estudios Fronterizos, 20, 1-28. https://doi.org/10.21670/ref.1901022.

Villalta, M. A. y Assael, C. (2018). Contexto socioeconómico, práctica pedagógica y aprendizaje autónomo en el aula. Estudios Pedagógicos, 44(1), 49-68. http://dx.doi.org/10.4067/S0718-07052018000100049

Walsh, C. (2014). ¿Interculturalidad? Fantasmas, fantasías y funcionalismos. Revista nuestrAmérica, 2(4), 17-20. http://www.revistanuestramerica.cl/ojs/index.php/nuestramerica/article/view/72/143 